International Mathematical Forum, 1, 2006, no. 27, 1313 - 1323

\title{
On The Sobolev-type Inequality for Lebesgue Spaces with a Variable Exponent
}

\author{
B.CEKIC ${ }^{a, 1}$, R.MASHIYEV ${ }^{a}$ and G.T.ALISOY ${ }^{b}$
}

${ }^{a}$ Dicle University, Dept. of Mathematics, 21280, Diyarbakır, Turkey

${ }^{b}$ Inonu University, Dept. of Mathematical Sciences, 44280, Malatya, Turkey

\section{Abstract}

This paper gives Sobolev-type inequality for the generalized Lebesgue space $L^{p(x)}(\Omega)$ and corresponding Sobolev space $W^{1, p(x)}(\Omega)$, consisting of all $f \in L^{p(x)}(\Omega)$ with first-order distributional derivatives in $L^{p(x)}(\Omega)$, where $\Omega$ is a bounded domain $R^{N}(N \geqslant 2)$, and $p(x)$ and $q(x)$ measurable function with $1 \leqslant q^{-} \leqslant q(x) \leqslant q^{+}<\infty, 1 \leqslant p^{+}<N$ and

$$
\sup _{x \in \Omega} q(x)\left[\frac{1}{p(x)}-\frac{1}{N}\right] \leqslant 1-\frac{\left(q^{+}-q^{-}\right)(N-1)}{N} .
$$

In other words, our aim in this study is obtained pre-limit condition for measurable variable exponent functions in Sobolev-type inequality.

Mathematics Subject Classification: 46E35, 26D10

Keywords: Variable exponent, Sobolev-type inequality

\section{Introduction}

Let $\Omega \subset R^{N}$ be an open set and $p: \Omega \rightarrow[1, \infty)$ be measurable function. We define the variable exponent Lebesgue spaces $L^{p(x)}(\Omega)$ consisting of all measurable functions $f: \Omega \rightarrow R$ such that

$$
\int_{\Omega}|\lambda f(x)|^{p(x)} d x<\infty
$$

for some $\lambda>0$. We can introduce the norm on $L^{p(x)}(\Omega)$ by

$$
\|f\|_{p(x), \Omega}=\inf \left\{\lambda>0: \int_{\Omega}\left|\frac{f(x)}{\lambda}\right|^{p(x)} d x \leqslant 1\right\}
$$

and the $L^{p(x)}(\Omega)$ becomes a Banach spaces [5].

\footnotetext{
${ }^{1}$ Correspondig Authour : bilalc@dicle.edu.tr This research is supported by the DUAPK 04-FF-40.
} 
The corresponding Sobolev space $W^{1, p(x)}(\Omega)$ is the class of all functions $f \in L^{p(x)}(\Omega)$ such that all generalized $D_{i} f, i=1,2, \ldots, n$, belong to $L^{p(x)}(\Omega)$. Endowed with the norm

$$
\|f\|_{1, p(x)}=\|f\|_{p(x)}+\|\nabla f\|_{p(x)}
$$

it forms a Banach space.

O.Kovác and J. Rákosník [5] obtained Sobolev-type inequality

$$
\|u\|_{q(x), \Omega} \leqslant c\|\nabla u\|_{p(x), \Omega} ; u \in C_{0}^{\infty}(\Omega) .
$$

for continuous $p(x)$ function, where a positive constant $c$ is independent of $u$ and

$$
1 \leqslant q(x) \leqslant \frac{N p(x)}{N-p(x)}-\varepsilon=p^{*}(x)-\varepsilon, \quad 0<\varepsilon<\frac{1}{N-1}
$$

and they showed that, in general, the Sobolev space $W^{1, p(x)}(\Omega)$ is not embedding in $L^{p^{*}(x)}(\Omega)$. Obviously, $q(x)=\frac{N p(x)}{N-p(x)}$ equality is not valid for above embedding when $p(x)$ is discontinuous. Naturally, $q(x)$ must be sufficiently less than $\frac{N p(x)}{N-p(x)}$.

Later D.E.Edmunds and J.Rákosník $[2,3]$ proved $W^{1, p(x)}(\Omega) \rightarrow L^{p^{*}(x)}(\Omega)$ for bounded Lipschitz domain, where $p(x) \in C^{0,1}(\bar{\Omega})$ and $1 \leqslant p(x) \leqslant q<N$.

X.L.Fan, J.Shen and D.Zhao [4] investigated Sobolev-type inequality for domain with cone property.

Recently, L.Diening [1] obtained above embedding for unbounded domain assuming that $p(x)$ is constant at infinity and satisfies (w-Lip.)

$$
|p(x)-p(y)| \leqslant \frac{C}{-\log |x-y|} \quad ; x, y \in R^{N},|x-y| \leqslant \frac{1}{2} .
$$

That is, under mentioned conditions, Sobolev-type embedding is proved in case of limit by L.Diening [1]. S.Samko [6] also found the following result for measurable variable exponent functions in generalized Lebesgue space:

Theorem 1.1 [6] Let $k(x) \in L^{Q}(B(0,2 D))$ where $Q \geqslant 1$ and $D=\operatorname{diam} \Omega$. The convolution operator

$$
K_{\Omega} f=\int_{\Omega} k(x-y) f(y) d y
$$

is bounded from $L^{p(x)}(\Omega)$ into $L^{r(x)}(\Omega), r(x) \geqslant 1$, if

$$
\frac{1}{Q} \leqslant 1-\frac{1}{p^{-}}+\frac{1}{p^{+}}, \quad \frac{1}{r(x)} \geqslant \frac{1}{Q}+\frac{1}{p^{+}}-1 .
$$


In this article, our aim is to show Sobolev-type inequality (1) for Lebesgue spaces with a variable exponent where $\Omega$ is assumed to be a bounded domain in $R^{N}$ and $p(x)$ is a measurable real-valued function (may be discontinuous) defined on $\Omega$. That is, we determine "Pre-limit" condition for measurable variable exponent functions in Sobolev-type inequality.

\section{Notation:}

In this study, $|\Omega|$ is the Lebesgue measure of a set $\Omega$ in $R^{N}$ and

$$
g^{+}=\sup _{x \in \Omega} g(x), g^{-}=\inf _{x \in \Omega} g(x), \delta=\frac{q^{+}-q^{-}}{N^{*}} \text { and } N^{*}=\frac{N}{N-1} \text {. }
$$

\section{A Sobolev-type Inequality}

Theorem 2.1: Let $\Omega \subset R^{N},(N \geqslant 2)$ be a bounded domain. Let $1 \leqslant q^{-} \leqslant q(x) \leqslant q^{+}<\infty$ and $1 \leqslant p^{+}<N$ be such that

$$
\sup _{x \in \Omega} q(x)\left[\frac{1}{p(x)}-\frac{1}{N}\right] \leqslant 1-\delta
$$

for $x \in \Omega$. Then, there exist a constant $C(N, p, q)>0$ such that

$$
\|u\|_{q(x), \Omega} \leqslant C(N, p, q, \Omega)\|\nabla u\|_{p(x), \Omega} \quad ; u \in C_{0}^{\infty}(\Omega),
$$

where

$$
C(N, p, q, \Omega)= \begin{cases}C(N, p, q)|\Omega|^{\frac{1}{N}-\frac{1}{p^{-}}+\frac{1}{q^{+}}} & \text {if }|\Omega| \leqslant 1 \\ C(N, p, q)|\Omega|^{\frac{1}{N}-\frac{1}{p^{+}}+\frac{1}{q^{-}}} & \text {if } \quad|\Omega|>1\end{cases}
$$

Proof: We suppose that $\|u\|_{q(x), \Omega}=1$ and $|\Omega|=1$. We have

$$
\begin{aligned}
1=\int_{\Omega}|u(x)|^{q(x)} d x & =\int_{E_{2}}|u(x)|^{q(x)} d x+\int_{E_{0} / E_{2}}|u(x)|^{q(x)} d x \\
& =I_{1}+I_{2},
\end{aligned}
$$

where $\mathrm{E}_{t}=\{x \in \Omega:|u(x)|>t\}$. Then it is easy to see that

$$
I_{2}=\int_{E_{0} / E_{2}}|u(x)|^{q(x)} d x \leqslant 2^{q^{+}-1} \int_{\Omega}|u(x)| d x
$$

and since

$$
|u(x)|^{q(x)}-1 \geqslant \frac{1}{2}|u(x)|^{q(x)}
$$


for $x \in E_{2}$, we can write

$$
I_{1}=\int_{E_{2}}|u(x)|^{q(x)} d x \leqslant 2 \int_{E_{2}} d x\left(\int_{1}^{|u(x)|} q(x) t^{q(x)-1} d t\right) .
$$

Also by Fubini's Theorem, we obtain

$$
I_{1} \leqslant 2 \int_{1}^{\infty} d t\left(\int_{E_{t}} t^{q(x)-1} q(x) d x\right) .
$$

By using Taylor's expansions of $e^{x}$, from (4) we get

$$
\begin{aligned}
I_{1} & \leqslant 2 \int_{1}^{\infty} t^{a-1} d t\left(\int_{E_{t}} t^{q(x)-a} q(x) d x\right) \\
& =2 \int_{1}^{\infty} t^{a-1} d t\left(\int_{E_{t}}\left(\sum_{n=0}^{\infty} \frac{(q(x)-a)^{n} q(x) \ln ^{n} t}{n !}\right) d x\right) \\
& =2 \int_{1}^{\infty} t^{a-1} d t\left(\sum_{n=0}^{\infty} \frac{\ln ^{n} t}{n !} \int_{E_{t}} q(x)(q(x)-a)^{n} d x\right),
\end{aligned}
$$

where $a \in R^{1}, a<q^{-}$. On the other hand, by means of Gagliardo's inequality [7], for $f \in C_{0}^{\infty}(\Omega), w \in L_{l o c}^{1}(\Omega)$

$$
\left(\int_{\Omega}|f|^{N^{*}} w(x) d x\right)^{\frac{1}{N^{*}}} \leqslant C(N) \int_{\Omega}|\nabla f| w(x)^{\frac{1}{N^{*}}} d x
$$

we obtain

$$
\begin{aligned}
& \left(\int_{\Omega} q(x)(q(x)-a)^{n}|f|^{N^{*}} d x\right)^{\frac{1}{N^{*}}} \\
& \quad \leq K^{\frac{1}{N^{*}}}(n, a)\left(\int_{\Omega}|\nabla f|(q(x)-a)^{\frac{n}{N^{*}}} q(x)^{\frac{1}{N^{*}}} d x\right)
\end{aligned}
$$

where

$$
K(n, a)=C(N)\left(\frac{q^{+}-a}{q^{-}-a}\right)^{n} \quad, n=0,1,2, \ldots
$$


If we take $Z(x)=\min \left(\frac{|u(x)|-\frac{t}{2}}{\frac{t}{2}}, 1\right), t>0$ in place of the test function $f$ in (7) then, we find

$$
\begin{aligned}
& \int_{E_{t}} q(x)(q(x)-a)^{n} d x \\
& \quad \leq \frac{K(n, a)}{t^{N^{*}}}\left(\int_{G_{t}}|\nabla u|(q(x)-a)^{\frac{n}{N^{*}}} q(x)^{\frac{1}{N^{*}}} d x\right)^{N^{*}}
\end{aligned}
$$

where

$$
G_{t}=\left\{x \in \Omega: \frac{t}{2}<|u(x)| \leqslant t\right\} .
$$

Hence, from ( 5 ) and ( 8 ) we have

$$
\begin{aligned}
I_{1} \leqslant 2 & \int_{1}^{\infty} t^{a-1} d t \times \\
& \left(\sum_{n=0}^{\infty} \frac{K(n, a) \ln ^{n} t}{n ! t^{N^{*}}}\left(\int_{G_{t}}|\nabla u|(q(x)-a)^{\frac{n}{N^{*}}} q(x)^{\frac{1}{N^{*}}} d x\right)^{N^{*}}\right) .
\end{aligned}
$$

By using Minkowski's inequalities for sums, we obtain

$$
\begin{aligned}
& I_{1} \leqslant 2 \int_{1}^{\infty} t^{a-1-N^{*}} d t \times \\
& \quad\left(\int_{G_{t}}|\nabla u| q(x)^{\frac{1}{N^{*}}}\left[\sum_{n=0}^{\infty} \frac{K(n, a) \ln ^{n} t}{n !}(q(x)-a)^{n}\right]^{\frac{1}{N^{*}}} d x\right)^{N^{*}} \\
& =2 C^{N^{*}}(N) \int_{1}^{\infty} t^{a-1-N^{*}} d t\left(\int_{G_{t}}|\nabla u| q(x)^{\frac{1}{N^{*}}} t^{\frac{(q(x)-a) \beta(a)}{N^{*}}} d x\right)^{N^{*}},
\end{aligned}
$$

where $\beta(a)=K^{\frac{1}{n}}(n, a)$. Again by using Minkowski's inequality for inte- 
grals we have

$$
\begin{aligned}
I_{1} \leq & 2 C^{N^{*}}(N) \times \\
& \left(\int_{E_{1}}|\nabla u| q(x)^{\frac{1}{N^{*}}} d x\left(\int_{|u(x)|}^{2|u(x)|} t^{(q(x)-a) \beta(a)+a-1-N^{*}} d t\right)^{\frac{1}{N^{*}}}\right)^{N^{*}} \\
\leq & \frac{2 q^{+} C^{N^{*}}(N)\left(2^{\frac{\left(q^{+}-a\right) \beta(a)}{N^{*}}+\frac{a}{N^{*}}-1}-1\right)}{\left(q^{-}-a\right) \beta(a)+a-N^{*}} \times \\
& \left(\int_{E_{1}}|\nabla u||u|^{\frac{(q(x)-a) \beta(a)}{N^{*}}+\frac{a}{N^{*}}-1} d x\right)^{N^{*}}
\end{aligned}
$$

From ( 3 ) and ( 10$)$, we get

$$
\begin{aligned}
1= & I_{1}+I_{2} \\
\leqslant & C\left(N, q^{+}, q^{-}, a\right)\left(\int_{E_{1}}|\nabla u||u|^{\frac{(q(x)-a) \beta(a)}{N^{*}}+\frac{a}{N^{*}}-1} d x\right)^{N^{*}} \\
& +2^{q^{+}-1} \int_{\Omega}|u| d x .
\end{aligned}
$$

and for $a \rightarrow-\infty$,

$$
\begin{gathered}
\frac{(q(x)-a) \beta(a)}{N^{*}}+\frac{a}{N^{*}}-1 \rightarrow \frac{q(x)}{N^{*}}-1+\frac{q^{+}-q^{-}}{N^{*}} \\
C\left(N, q^{+}, q^{-}, a\right) \rightarrow C\left(N, q^{+}, q^{-}\right)
\end{gathered}
$$

where $C\left(N, q^{+}, q^{-}\right)$is finite. If we apply Fato's Lemma to ( 11$)$, then we write

$$
1 \leqslant C\left(\int_{E_{1}}|\nabla u||u|^{\frac{q}{N^{*}}-1+\delta} d x\right)^{N^{*}}+2^{q^{+}-1}\left(\int_{\Omega}|u|^{N^{*}} d x\right)^{1 / N^{*}}
$$

where $C>0$ only depends on $N, q^{+}$and $q^{-}$.

If we apply Young inequality $d b \leqslant \varepsilon d^{p}+c(\varepsilon) b^{p^{\prime}} ; d>0, b>0, \varepsilon>0, p>1$ to first term of inequality ( 12 ) and inequality ( 6 ) to second term for $w(x)=1$, 
we obtain

$$
\begin{aligned}
1 \leqslant & C\left(C(\varepsilon) \int_{\Omega}|\nabla u|^{p(x)} d x+\varepsilon \int_{\Omega}|u|^{\left(\frac{q}{N^{*}}-1+\delta\right) p^{\prime}(x)}\right)^{N^{*}} \\
& +2^{q^{+}-1} \int_{\Omega}|\nabla u| d x,
\end{aligned}
$$

where $p(x): \frac{1}{p(x)}+\frac{1}{p^{\prime}(x)}=1,1 \leqslant p(x)<\infty$.

By assumption of theorem, we have

$$
\left[\frac{q(x)}{N^{*}}-1+\delta\right] p^{\prime}(x) \leqslant q(x)
$$

and by using Young's inequality again to last integral in the inequality (13), then we write

$$
1 \leqslant C\left[C(\varepsilon) \int_{\Omega}|\nabla u|^{p(x)} d x+\varepsilon\right]^{N^{*}}+C(\varepsilon) \int_{\Omega}|\nabla u|^{p(x)} d x+\varepsilon 2^{q^{+}-1} .
$$

Hence, for sufficiently small $\varepsilon>0$ we obtain

$$
1 \leqslant C\left[\int_{\Omega}|\nabla u|^{p(x)} d x+\left(\int_{\Omega}|\nabla u|^{p(x)} d x\right)^{\frac{1}{N^{*}}}\right] .
$$

If $C\left(\int_{\Omega}|\nabla u|^{p(x)} d x\right)^{\frac{1}{N^{*}}}<\frac{1}{2}$, then we have from (14)

$$
1 \leqslant C \int_{\Omega}|\nabla u|^{p(x)} d x
$$

To contrary, if $C\left(\int_{\Omega}|\nabla u|^{p(x)} d x\right)^{\frac{1}{N^{*}}} \geqslant \frac{1}{2}$, it follows that $2 C \int_{\Omega}|\nabla u|^{p(x)} d x \geqslant 1$. Therefore we have $2 C\left(\int_{\Omega}|\nabla u|^{p(x)} d x\right)^{\frac{1}{N^{*}}} \leqslant(2 C)^{N^{*}}\left(\int_{\Omega}|\nabla u|^{p(x)} d x\right)$ and from ( 14 ) we can obtain inequality ( 15 ) again.

Now we suppose that $\|u\|_{q(x), \Omega}$ is any number. Then, inequality (15) for function

$$
z=\frac{u}{\|u\|_{q(x), \Omega}}
$$


follows that $1 \leqslant C \int_{\Omega}\left(\frac{|\nabla u|}{\|u\|_{q(x), \Omega}}\right)^{p(x)} d x$. Hence,

$$
\|u\|_{q(x), \Omega} \leqslant C(N, p, q)\|\nabla u\|_{p(x), \Omega} .
$$

We suppose that $|\Omega|$ is any positive number. Then we consider the mapping $x:=T y=x_{0}+r y$, where $r>0, x_{0} \in \Omega$ and $x_{0}$ is fixed. It is easy to see that range of $\Omega$ is $\Omega^{*}$ as $x \rightarrow y$. We assume that $\widetilde{u}(y)=u\left(x_{0}+r y\right)$, $\widetilde{p}(y)=p\left(x_{0}+r y\right)$ and $\widetilde{q}(y)=q\left(x_{0}+r y\right)$. We choose $r$ such that $\left|\Omega^{*}\right|=1$. i.e. $r=C_{N}|\Omega| \frac{1}{N}$.

By the definition of norm, we obtain $\int_{\Omega^{*}}\left(\frac{|\widetilde{u}(y)|}{\|u\|_{\tilde{q}(y), \Omega^{*}}}\right)^{\widetilde{q}(y)} d y=1$. If $|\Omega| \leqslant 1$, by using inverse mapping we obtain

$$
\int_{\Omega}\left(\frac{|u(x)|}{\|\widetilde{u}\|_{\widetilde{q}(y), \Omega^{*}}|\Omega|^{1 / q^{+}}}\right)^{q(x)} d x \leqslant 1 .
$$

Therefore,

$$
\|u\|_{q(x), \Omega} \leqslant C_{N}|\Omega|^{\frac{1}{q^{+}}}\|\widetilde{u}\|_{\widetilde{q}(y), \Omega^{*}} .
$$

Again, with respect to definition of the norm of gradient of function, we find

$$
\int_{\Omega}\left(\frac{|\nabla u|}{\|\nabla u\|_{p(x), \Omega}}\right)^{p(x)} d x=1 .
$$

Then, we have

$$
|\Omega| \int_{\Omega^{*}}\left(\frac{|\nabla \widetilde{u}|}{r\|\nabla u\|_{p(x), \Omega}}\right)^{\tilde{p}(y)} d y \leqslant 1 .
$$

and

$$
\int_{\Omega^{*}}\left(\frac{|\nabla \tilde{u}|}{|\Omega|^{\frac{1}{N}-\frac{1}{p^{-}}}\|\nabla u\|_{p(x), \Omega}}\right)^{\tilde{p}(y)} d y \leqslant 1
$$

where $|\Omega| \leqslant 1$.

As a result of ( 18 ), we obtain

$$
\|\nabla \widetilde{u}\|_{\widetilde{p}(y), \Omega^{*}} \leqslant|\Omega|^{\frac{1}{N}-\frac{1}{p^{-}}}\|\nabla u\|_{p(x), \Omega} .
$$


From ( 16$),(17)$ and ( 19$)$ for $u$, we have

$$
\|u\|_{q(x), \Omega} \leqslant C(N, p, q)|\Omega|^{\frac{1}{N}-\frac{1}{p^{-}}+\frac{1}{q^{+}}}\|\nabla u\|_{p(x), \Omega},
$$

where $|\Omega| \leqslant 1$.

As similarly,

$$
\|u\|_{q(x), \Omega} \leqslant C(N, p, q)|\Omega|^{\frac{1}{N}-\frac{1}{p^{+}}+\frac{1}{q^{-}}}\|\nabla u\|_{p(x), \Omega}
$$

where $|\Omega|>1$. The theorem is proved.

\section{Remark}

Let $D$ and $E$ be any regular bounded domains in $R^{2}$ and $E \subset D$. Consider functions

$$
p(x)=\left\{\begin{array}{lll}
4 / 3 & \text { if } & x \in E \\
1 & \text { if } \quad x \in D / E
\end{array}\right.
$$

and

$$
q(x)=\left\{\begin{array}{lll}
2 & \text { if } & x \in E \\
1 & \text { if } & x \in D / E
\end{array} .\right.
$$

It is clear that conditions of Theorem 1.1 do not hold in this case. But Theorem 2.1 holds.

Theorem 2.2. Let $\Omega \in R^{N}$ be an open bounded set with Lipschitz boundary, $p(x) \in w-$ Lip. and $1<p^{-} \leqslant p^{+}<N$. Then, for all measurable $q: \Omega \rightarrow[1, \infty)$ with $q(x) \leqslant p^{*}(x)-\delta \quad\left(p^{*}=\frac{N p(x)}{N-p(x)}\right)$ and some $\delta>0$, there holds $W^{1, p(.)}(\Omega) \rightarrow \rightarrow L^{q(.)}(\Omega)$. i.e. the embedding is compact.

Proof: There exist $m$ such that $q \leqslant m \leqslant p^{*}$, where $0<p(p-m)<$ $m\left(p-p^{*}\right)$. Note that $m$ is a bounded exponent. Let $u_{n}, u \in W^{1, p(.)}(\Omega)$ with $u_{n} \rightarrow u$ (weak $\left.\lim \right)$. We have to show that $u_{n} \rightarrow u$ in $L^{q(.)}(\Omega)$ (strong lim). Theorem 2.1 implies chains of embeddings

$$
W^{1, p(x)} \rightarrow W^{1, m(x)} \rightarrow W^{1, m^{-}} \rightarrow L^{\left(m^{-}\right)^{*}} \rightarrow L^{q^{*}} \rightarrow L^{q(x)} \quad,\left(m^{-}\right)^{*} \geqslant q^{*}
$$

and from the generalized Hölder's inequality [1], we obtain

$$
\left\|u_{n}-u\right\|_{m} \rightarrow 0
$$

or

$$
u_{n} \rightarrow u
$$


in $L^{q(\cdot)}(\Omega)$.

Now, we consider of the classes

$$
\|u\|_{W_{x_{j}}^{m, p(x)}(\Omega)}=\|u\|_{L^{p(x)}(\Omega)}+\left\|\frac{\partial^{m} u}{\partial x_{j}^{m}}\right\|_{L^{p(x)}(\Omega)}, \quad(j=1, \ldots, n) .
$$

Theorem 2.3. Let $\Omega_{k|h|} \subset \subset \Omega$ and $k h<\operatorname{dist}\left(\Omega_{k|h|}, \partial \Omega\right)$, if $u \in W_{x_{j}}^{k, p(x)}(\Omega)$ where $1 \leqslant p^{-} \leqslant p^{+}<\infty$ and $p(x) \in w-L i p$., then $\Delta_{x_{j}, h}^{k} u(x) \in L^{p(x)}\left(\Omega_{k|h|}\right)$ and we have

$$
\left\|\Delta_{x_{j}, h}^{k} u\right\|_{p(x),\left(\Omega_{k|h|}\right)} \leqslant C_{k}\left\|\frac{\partial^{k} u}{\partial x_{j}^{k}}\right\|_{p(x),(\Omega)}
$$

Proof. For $k=1,\left(x_{1}, \ldots, x_{n}\right) \in \Omega_{|h|}$ and $\Delta_{x_{1, h}} u(x)=\int_{0}^{h} u_{x_{1}}^{\prime}\left(x_{1}+t, x_{2}, \ldots, x_{n}\right) d t$, we can write

$$
\begin{aligned}
\left\|\Delta_{x_{1, h}} u\right\|_{p(x),\left(\Omega_{|h|}\right)} & \leqslant\left|\int_{0}^{h}\left\|u_{x_{1}}^{\prime}\left(x_{1}+t, x_{2}, \ldots, x_{n}\right)\right\|_{p(x),\left(\Omega_{|h|}\right)} d t\right| \\
& \leqslant\left|\int_{0}^{h}\left\|u_{x_{1}}^{\prime}\right\|_{p(x),(\Omega)} d t\right| \\
& =C\left\|u_{x_{1}}^{\prime}\right\|_{p(x),(\Omega)} .
\end{aligned}
$$

Therefore, for any $k$ we obtain

$$
\begin{aligned}
\left\|\Delta_{x_{j, h}}^{k} u\right\|_{p(x),\left(\Omega_{k|h|}\right)} & =\left\|\Delta_{x_{j}, h}\left(\Delta_{x_{j, h}}^{k-1} u\right)\right\|_{p(x),\left(\Omega_{k|h|}\right)} \\
& \leqslant C_{1}\left\|\Delta_{x_{j, h}}^{k-1} u_{x_{j}}^{\prime}\right\|_{p(x),\left(\Omega_{(k-1)|h|}\right)} \\
& \cdot \\
& \cdot \\
& \leqslant C_{k}\left\|u_{x_{j}}^{(k)}\right\|_{p(x),(\Omega)} .
\end{aligned}
$$

Theorem 2.3. is proved

ACKNOWLEDGEMENTS.The authors would like to thank S.G.Samko for a useful discussion. 


\section{References}

[1] L.Diening, Riesz potential and Sobolev embeddings of generalized Lebesgue and Sobolev spaces $L^{p(x)}(\Omega)$ and $W^{k, p(x)}(\Omega)$. Math. Nachr., October 8, 2003, 112-115.

[2] D.E. Edmunds, J. Rákosník, Sobolev embedding with variable exponent. Studia Math., 143 no.3 2000, 267-293.

[3] D.E. Edmunds, J. Rákosník, Sobolev embedding with variable exponent II. Math.Nachr., 246-247, 2002, 53-67.

[4] X.L. Fan, J.S. Shen, D. Zhao, Sobolev embedding theorems for spaces $W^{k, p(x)}(\Omega)$. J.Math. Anal. Appl., 262, 2001, 749-760.

[5] O. Kovác, J. Rákosník,On spaces $L^{p(x)}(\Omega)$ and $W^{k, p(x)}(\Omega)$.Czechoslovak Math.J., 116 1991, 592-618.

[6] S.G. Samko, Convolution and potential type operators in $L^{p(x)}\left(R^{n}\right)$. Integr. Transform. and Special Funct. v.7, 3-4 1998, 261-284.

[7] E. Sawyer, R.L. Wheeden, Weighted inequalities for Fractional Integrals on Euclidian and Homogeneous Spaces.American Journal of Math., 114, $1992,813-874$.

Received: October 14, 2005 